\title{
Presumed Cause(s) of Tuberculosis in Rural Nepal
}

\section{2) Ulla-Britt Engelbrektsson \\ Ladhusudan Subedi}

\begin{abstract}
In focus is the emic perspective on cause/s of tuberculosis. The ethnographic data is from two major studies in one and the same community in the Hill district of Pyuthan, in the Mid-Western Development Region. The findings show a pooling of old and new influences with a dazzling plurality of thoughts and ideas within a framework of great uncertainty. Moreover, changes in perceptions over the last decade and an half are quite marginal. For health workers to function efficiently the perceived causes of patients and their communities need to be understood and taken into account.
\end{abstract}

Keywords: Explanatory Model, Tuberculosis, Presumed Cause/s

\section{Introduction}

In Nepal, approximately 40,000 annually develop active tuberculosis (TB) and 5,000-7,000 die from the disease. Biomedically, TB is an infectious disease, in humans mainly caused by Mycobacterium Tuberculosis. The disease usually affects the lungs (pulmonary TB) but can also occur in other parts of the body (extra-pulmonary TB). Approximately half of those who develop the disease are potentially infectious. Those are patients with sputum-smear positive pulmonary TB. Extra-pulmonary TB is virtually non-infectious. When a person with infectious TB coughs, sneezes, spits or talks, droplets containing TB bacteria are released into the air. A person needs to inhale only a few to become infected. Exposure, however, does not necessarily result in infection. Moreover, an infection may progress to an active disease process or the bacteria stay dormant in the body in a condition known as latent tuberculosis. Dormant TB organisms do not infect others. Conditions which compromise the immune response, such 
as a TB-HIV, predispose to the development of active TB. Yet, only one in ten with latent tuberculosis is likely to develop active TB. Presently, active drug-sensitive TB is treated with a standard sixmonth course of four antimicrobial drugs. Almost 95 per cent of the bacterial sterilisation takes place within the first two weeks of the therapy (Dheda, 2010, p.17).

There are a number of excellent studies of various aspects of TB in Nepal. Outstanding in regard to the topic at hand is a study carried out by Bhatt and co-researchers in 2006, reported on for instance in the SAARC Journal of Tuberculosis, Lung Diseases and HIV/Aids in 2009, under the title "Nepalese People's knowledge about Tuberculosis". The study found that the patients' knowledge about causative factors was low. There is also the large scale KAP study on Tuberculosis and TB/HIV Co-infection carried out by Health Research and Development Forum in ten districts across Nepal which concludes that 80 per cent of the community people, TB-infected people, and their family members did not know about the main causative agent of TB. This article is a complementary addition, a social anthropological study of perceptions over time within one and the same community, combined with an attempt to relate particulars to a wider Nepali context and to the biomedical model of TB.

\section{The Theoretical and Methodological Approach}

Causality can be identified and understood on different levels. Thus, as demonstrated by Evans-Pritchard in his Azande material in 1937, belief in witchcraft does not necessarily contradict empirical knowledge of cause and effect. Witchcraft answers the question of "why" misfortune happens to a particular person but not necessarily "how" it happens. Salzberger (1976) proposes that, generally speaking, the responsibility for an illness is ascribed either to the victim or to external factors. In the first group of hypotheses, the individual is made responsible for his/her choices, conscious or unconscious, and hence for his/her sickness. In the second group the individual is thought of as a victim of chance. Foster and Anderson (1978) differentiate between personalistic and naturalistic aetiologies. The former is a system in which illness is believed to 
be caused by the active, purposeful intervention of a sensate agent, whereas the latter is a construction in which illness is explained in impersonal, systemic terms. They suggest that a combination of personalistic and naturalistic explanations is common in medical systems, but with one usually much favoured over the other.

A useful tool for analysing therapeutic episodes is the concept of the Explanatory Model (EM) developed by Kleinman (1980). EMs are held by both patients and practitioners. The aetiology (the history and presumed cause/s) of a condition is one aspect of an EM. Kleinman suggests, that whereas practitioners' EMs are mostly based on a single causal train of logic, lay EMs tend to be idiosyncratic and changeable and influenced by both personality and cultural factors. Young (1992) adds that a wide array of fragmentary explanations from multiple sources and different knowledge structures may be used in the construction of lay illness narratives. And Helman (2007) suggests that in most cases, lay theories of illness aetiologies are multi-causal, that is, they postulate that several causes act together.

Two major field-studies, each running over a month and an half, were carried out in one and the same community, in Solubang, the first in 2005 and the second in 2013/14. In both instances TB patients from the preceding seven years were in focus. Besides the patients, one household member and one next-door neighbour of each patient were interviewed (120 formal interviews in total). Moreover, there were observations and informal talks with other community members and with local health workers. In addition, in the second study there were follow-up interviews with the patients of the first study. Responses were analysed thematically.

\section{Key Findings}

The agricultural village of Solubang is populated by several castes but with Chhetries in the numerical and political lead. The TB patients were found by asking around and by help of TB registers and treatment cards at local and nearby DOTS centres. In 2005, 24 Solubang TB patients from the preceding seven years were thus identified. In 2013, the same procedures resulted in 15 patients. The caste distribution reflected that of the village as a whole. There were 
24 males versus 13 females (close to the national TB M/F ratio).

Before the availability of anti-TB medicines, TB was a much feared condition in Solubang, as in the rest of Nepal. The local denotation was 'khapte' a word used for half-rotten fruit (the other half also likely to rot) and/or 'sukenashak' meaning thin/wasted. The condition was believed to be transmittable to others. Those in advanced stages of the disease were put out of the house. When possible, they were placed under a pine tree as such trees were believed to have a soothing and perhaps even a healing influence, even so, most died within a short period of time. Later the medical Nepali term for tuberculosis, 'kshayarog' was adopted and still later the English abbreviation 'tibi' (TB).

\section{The first study, 2005}

At the time of the first study, the population of Solubang amounted to just fewer than 800 persons in approximately 130 households. Living quarters were clustered caste-wise. Only a quarter, it was said, had enough crops to last for a year. Thus, additional work had to be found. Some did so within the district as day labourers and in low-level government jobs and others in seasonal or long term labour in India. A few families had moved out but no one, it was said, had moved in, thus, it was a well-grounded population but with plenty of opportunities for influences from the outside.

The residents reckoned that the village had and had more than its fair share of TB. The spontaneously suggested reason was "too much alcohol".

In general when sick, the first choice outside the home was a consultation with a local dhami/jhankri (shaman) of one's own caste, the second a more powerful dhami/jhankri of any caste within or out of the village. At the same time there were government health facilities nearby: the district hospital south-west of the village and a health post south-east of it. Their services were well thought of but were usually not the first port of call.

In 2005, as mentioned, 24 TB patients were identified within a 7-year cohort. Twenty-two of them (14 males, 8 females) were registered for treatment within the district, with somewhat less than half diagnosed as sputum-smear positive. 


\section{The neighbours}

Most respondents answered well in regard to signs and symptoms of pulmonary TB. This was the case of an elderly man who said, "We know it is TB when someone starts to cough, bleed from the mouth, not feel like eating, have fever in the evening, and the person loses weight". A few added, "The face gets dark". Only one or two, referred to a sign or symptom specifically connected with extra-pulmonary TB. Yet, 6 of the 24 patients in the cohort were registered as such.

In response to a general question about the cause/s of $\mathrm{TB}$, a few suggested that they did not know, some mentioned one or two possibilities, but most proposed several ideas. Even so, a common denominator was that the disease was "from the person's own body", that is, a condition not caused by supernatural forces within the shamanistic sphere. Moreover, it was frequently stressed that it was from "a weak body", a body weakened by poverty often in connection with too much alcohol. A few added that TB was likely to be "hereditary". "Germs" were mentioned by one or two but not emphasised. Child-TB was not referred to at all. Yet, 6 of the 24 patients within the cohort were such cases.

Besides the general query about cause/s we asked who they knew who had or had had TB and their opinion about the cause/s in each case. With rare exceptions the replies can be summed up in: "They had this disease because a lack of cleanliness, carelessness with their eating habits, and because of drinking a lot of alcohol and smoking". Only one or two spontaneously suggested that the cause was likely to be a transmission from one person to another. Even so, when asked, "Is TB transmittable?" almost all acknowledged this to be the case.

A transmission, it was said, was possible by sharing food, coughing, sleeping together, and by sitting next to a person with TB. At the same time, the majority happily recognized that contrary to earlier days there was no longer a fear or hesitation to be close to a TB patient. In older days, it was said, TB patients were "hated" and avoided, some being moved out of the house and some even out of the village. However, this was no longer the case and should not be the case because "nowadays TB is a normal disease". The 
latter mostly explained as "a curable disease". In the same breath, in response to the question "Did/does the person isolate him/herself? If so, in what way?" almost all reported that the patient did so within his/her own household. Not by staying separate but by not giving his/her jutho (food remains) to others, by sleeping alone, having separate eating utensils, and by not sharing clothes with household members. Outside the household and in relation to others, however, there were no restrictions except not to eat the jutho, food from the patient's plate. There even seemed to be a certain pride in there being no restrictions. One or two, however, did express that, while outwardly happy when together with a TB patient, they were not necessarily completely at peace.

\section{The household-members}

Most started off by saying that they did not know the cause/s but would eventually present not one but several ideas. "Alcohol" was mentioned by a majority but most often not as the sole cause, particularly not in regard to the TB of the household member. "A weak body", as a main or the major cause was stressed by many. This was the case of a mother with a teenage son affected by TB, who explained that he had been weak and thin from birth onwards and this was because he did not eat well. Some located "the weakness of the body" to a specific occasion. This was the case of the widow of a man who died while on treatment, she reported, "Just before he got this disease there was a wedding in the village and at the occasion he drank a lot of alcohol and there was a fight and he was beaten badly, and afterwards he was very sick and also got TB". Yet, another respondent after having proposed "too much alcohol" simply added "...maybe he had also eaten something poisonous". One or two suggested that "having been in India" might have caused the condition.

When specifically asked, almost all the respondents, like the neighbours, acknowledged TB to be a transmittable disease. This was the case with a parent who proposed that his son's TB might have come about "...because he was familiar with a TB patient". Such a straightforward suggestion of the possibility of a transmission, however, was rare. Having acknowledged TB to be 
a transmittable disease most respondents simply left it at that. And yet, in several of the households/families there had been others with TB. Having shortly before acknowledged TB to be transmittable a young man in such a household suggested that perhaps the reason for his brother's disease was because the condition was "hereditary" as their mother had earlier had TB. In and through it all, there were many uncertainties.

\section{The patients}

So what about the patients themselves, what did they say? Again, there were various suggestions. When asked about the key cause the most common response was a direct or an indirect reference to "a weak body". Asked to elaborate upon the subject, most would say that their body was weak because of poverty, a lack of proper food, having worked too hard, alcohol, and so on. Again, some referred to or added specific circumstances. This was the case of a man who said, "While going near the stream, I might have been attacked by a god or while sleeping I had a wrong posture and got the chest pain". Another respondent suggested, "... and maybe because a dirty environment and by eating dirty things".

When specifically asked, all except one agreed to TB being a transmittable disease with one respondent restraining the possibility of transmission "to certain seasons". The one who did not agree to TB being transmittable was a female patient diagnosed as sputumsmear positive. Again, however, in sharing their own ideas of why they themselves ended up with TB, hardly anyone referred to the possibility of a transmission, and the few who did never suggested it as the only reason.

Most of them agreed to having heard that "germs" (kira/kiraunu/ biraunu) were involved: health workers had mentioned "germs" or they had heard it referred to over the radio, and so on. And, they themselves were absolutely willing to include germs as a cause but the concept was obviously not uppermost in their thinking.

To the question: "While on treatment, do/did you believe that you can/could give the disease to others. And if so how/by what route?" an older respondent, a pulmonary sputum-smear positive case did not reply but the rest of the adults, also the non-infectious 
cases (except one), testified to believing themselves to be or having been contagious. And, in most households, also in those with noninfectious patients, precautions against an internal transfer had been taken, in most cases instituted by the patient him- or herself. Moreover, such safety measures were commonly upheld for the whole treatment period and in some cases even longer.

\section{The second study, 2013/14}

Agriculture was continuously the main occupation in Solubang but the number and percentage of people employed outside had risen. Next to the village a tarmac road had been built which made it easier for the residents to reach the district headquarters but also other localities within and outside the district.

In 2013/14, there were approximately as many practising traditional healers as seven years earlier. They were routinely consulted but perhaps not as persistently as previously. Government health services were, as earlier, accessible and thought well of. The Health Post had been upgraded to a Primary Health Care Centre (PHCC). Even so, as before, in most cases the government health facilities were not the first port of call, not even when TB was suspected. The government health services were said to be good in treating TB but not in diagnosing the condition. Meanwhile, private pharmacies in the nearby district headquarters had multiplied and attracted a steadily growing number of Solubang customers.

\section{The previous cohort}

In between 2005-2013, four patients of the first cohort had died. According to family members, TB or the aftermath of TB was the major reason for three of the four deaths. Two had had a relapse. One of them, a rather young man told that quite soon after having been declared cured, he started to have similar symptoms as earlier. In 2013, when asked "why a relapse?" he replied, "I have a drinking and tobacco chewing habit, it might have been because of that". The second case, a woman in her thirties, also related her relapse to drinking and tobacco.

In 2013, the remaining patients of the 2005 cohort were asked how they presently perceived the cause/s of their disease. The 
suggestions of 2013 were not always the same as those proposed in 2005. Had we pinpointed the variances (which we did not) the respondents were likely to have suggested that the present suggestions did not necessarily rule out the earlier ones and vice versa. We also asked if, looking back, they thought that something could have been done to prevent them from getting the disease. One of the previous Child-TB cases said, “...perhaps if I had been vaccinated", the rest said, "No", even those who had previously suggested that drinking and smoking had been a main or the main cause of their TB.

\section{The new cohort}

In between 2005 to 2012/13, 15 Solubang residents were registered as TB patients (10 males and 5 females). Two-thirds were registered as pulmonary cases with half diagnosed as sputum-smear positive. This compared to 24 cases, or rather 22 locally registered cases, with half sputum-smear positive in the earlier cohort. The numbers, however, are not altogether comparable because the borders of the village had changed somewhat. Moreover, rumours had it that, in contrast to earlier, some Solubang TB patients, on purpose had or had had their treatment outside the district. Even so, epidemiologically there seemed to have been a slight improvement, which was further indicated by only one case being a Child-TB case. There had been no contact surveys.

More than half of the patients of the new cohort had a family member or a next-door neighbour previously affected by TB. When asked if family members had been for check-ups, the mother of a highly infectious youngster spontaneously replied, "We haven't been sick, so why should we have had a check-up?'”

In the 2012/13 cohort three had died before we re-entered the scene. One of them, a man in his sixties, had had a relapse. He died shortly before completing the second round of treatment. His widow had left for another village but the neighbours ascribed his death to "too much alcohol before, between, and during the treatments". Another patient, a woman in her mid-forties, had died shortly after the diagnosis. At the time of registration her weight was $32 \mathrm{~kg}$. The common explanation for her death was that she was not treated in 
time. The third who had died was an older woman. Her husband was among the patients of the previous cohort. He was the one who suggested that TB was only transmittable during specific seasons. In 2013, when asked about the cause/s of his wife's TB, he said, "At home I had TB and in my neighbourhood there were and are people with $\mathrm{TB}$, so it might have been transmitted to her from someone". He further reported that his wife had been well after the TB treatment but later got stomach problems and had died from her stomach problems.

\section{The neighbours}

When asked about the cause/s of TB in general, there was a wide assortment of suggestions. And, again as previously, transmissions were seldom amongst those spontaneously mentioned. Even so, when specifically asked, most agreed to the condition being transmittable, this time, it seemed, with a greater conviction than in 2005. Asked to illustrate such a process, one neighbour replied, "Some persons got TB from their neighbours, some while working in India, and some when working in the hospital". He added, "If one is careful about one's diet, one is less likely to get TB, but some have hereditary TB". Yet another respondent said, "The adults might get this disease from others but there are also children with this disease. I don't know why or how".

In regard to the cause/s of TB in identified individual cases several ideas were on offer. The most common, in no specific order were: drinking too much alcohol; lack of food; hereditary; bad/ unclean food and water; cigarettes/chewing-tobacco; transfers by sharing food and bed with a patient; from the person's own body; and many go to India to work and they bring this disease from there. Again, there were many 'hola' ('maybes'), that is: all within a framework of uncertainty. Thus, a large number of possible/ assumed causes were presented with few firm convictions except that alcohol, a weak body, and transmission were likely to be involved, sometimes all three, sometimes only one or two of them.

\section{The household-members}

Also this time, there were more than a few suggestions about 
the cause/s of TB in general and in relation to the TB of a family member. In addition, in several cases there were differences of opinions. The father of the only Child-TB case in the cohort, a boy of two and an half years of age at the time of the diagnosis, suggested, "He was treated for pneumonia for two months, perhaps that is why he got TB". The boy's mother said, "Maybe because he was born one month and a week too early". A middle-aged man suggested that he did not know the cause/s of TB because no one had told him but he had heard that it was connected with too much alcohol. His wife said the same and also disclosed that her husband drank a lot. The wife, but not the man, mentioned that before him their son had TB. While the wife and husband suggested too much drinking as a likely cause, others expressed a suspicion of a transfer of the disease from son to father, particularly as there had been no separation within the household. The son seemed to think the same. $\mathrm{He}$, a highly infectious case, told that after the diagnosis he had covered his mouth but only for a week. However, there were also agreements. This was the case with a middle-aged man diagnosed as a highly infectious case who said, "What I have understood is that this disease develops within one's own body". His brother said the same.

In and through it all, there were confusing situations. This obviously was the case in a family in which two sons of three had ended up with TB. The eldest was included in the 2005 study, the younger in the 2012/13 cohort. In 2013, when asked about the cause/s of TB in general their mother said, "I don't know. Maybe it comes from one's own body". She had good reasons not to be sure. In connection with the eldest son, diagnosed as an extra-pulmonary case (non-infectious), the health worker had said that his TB did not transfer and thus no need for precautions. They adhered to the advice. They did the same in the case of the younger son. The younger son, however, unlike his elder brother was a sputum-smear positive case. A year later, an elder brother of hers was diagnosed with TB. Meanwhile, the younger son suggested, "Maybe, I got my TB from my elder brother (the non-infectious one)".

Two-thirds of the patients within the cohort, as mentioned, were diagnosed as non-infectious cases. Even so, in most TB households, 
protective safety measures had been installed, again mostly on the initiative of the patient. And, commonly, again as within the 2005 cohort, the separation measures were upheld for the whole treatment period and in some cases even longer.

\section{The patients}

As in the earlier cohort, the patients presented several likely cause/s, both in regard to TB in general and to their own TB, with some seemingly quite at odds with each. Even so, approximately half suggested that the basic cause of TB was alcohol and cigarettes. At the same time, everyone knew that some TB patients had used neither alcohol nor tobacco. This was evidently the case of a girl who was only nine when diagnosed. At the time of the interview she was fifteen. To the question about the cause/s of TB in general and to her own TB in particular, she replied, "I think it might be because of smoking cigarettes or drinking alcohol, or maybe it is a hereditary disease, but there is no one in my family with a chronic disease. Some people also say that it transmits from sharing food. Maybe it came that way!" A minute later, she added, "My cousin had TB, that's why I think it might be hereditary, otherwise why should a child have TB?"

One of the patients, a Solubang man working as an office helper in the district hospital (treated without a clinic card), said that the doctor had told him about the cause of the disease. About the latter he said, "Maybe, the smoke, the dust, and the physical weakness are the causes of this disease". He added, "My fellow villagers have different opinions on this. Some think it is a hereditary disease whereas others think that drinking alcohol and smoking cigarettes are the causes". Somewhat later, he admitted, "Yes, it does transfer from one person to another. It transfers through breath and while sneezing. I have kept myself apart from my wife". We understood him to be a sputum-smear negative case, a non-infectious case. His next-door neighbour said, "Maybe he got TB because he works in the hospital. I have heard that this disease transfers from others and sometimes it develops from one's own body."

Again as in the previous study, when specifically asked if TB is a transmittable disease, almost all agreed to this being so. This 
was the case of a respondent in his forties, a sputum-smear negative case, who said, "I know that it transfers from one person to another, the others also know because we have all heard this on the radio." To the question about the cause/s in his own case, he replied, "There might be a cause/causes but I don't know because I am illiterate". This he said a minute after having said, "It depends upon one's body. Like my wife had chest problem and I had TB". A female respondent, a non-medical employee of the nearby PHCC, said, "I have heard that if a person drinks alcohol and smokes cigarettes or chews tobacco he/she will have TB". She added, "Yes, I can transfer this disease by my breath and by my spit". She also said, "After my marriage, I learnt that my mother-in-law had had TB". She did not say, "...most likely I picked it up at my place of work," a suggestion made, on her behalf, by her next-door neighbour.

There were three registered relapse cases within the cohort, all men. One of them was the man in his sixties, mentioned above, who had died shortly before completing his second round of treatment. The supposed 'relapse' of another man was more likely 'a return after defaulting'. His treatment was started in India but was not completed. In India, his brother before him had been treated for TB. His answer to the query about the cause/s of TB was, "They say that poverty and alcoholism are the causes". About himself he suggested, "Maybe it transferred to me from my neighbour or from family members". The third person was a patient interviewed already in 2005, a sputum-smear positive case. At the time of the first interview he was close to the end of his TB treatment. The medicines, he said, had definitely brought about a change for the better. Even so, half a year after having been declared cured, again there were severe chest pains. "I went to the hospital for a check-up but they could not tell what was wrong and therefore I went to India where my family is". Before that he had tried the dhami/jhankri, an astrologer, and had performed a number of religious rituals. In India he had more than one type of treatment. Having returned to Nepal, he was registered as a relapse case and was re-started on treatment. He, however, defaulted. "I went to an herbal doctor", he said. "The herbal doctor told me that if I drink one glass of alcohol per day for eight days I will get well. Eight days later, I revisited 
the herbal doctor and this time he advised me to drink two glasses of alcohol per day. I followed his instructions and I got well!" His elder brother told, "While on government treatment, both times, he stopped drinking and stopped chewing tobacco. But now he chews tobacco and drinks two glasses of alcohol per day."

\section{Discussion and Theoretical Implication}

The most outstanding feature in the ethnographic data is the great number of diverse causes suggested and the circumstance of the same person often proposing several causes, some of which appear to be quite contrary to one another. In addition, what is suggested at one time is not necessarily the same as the one/s proposed at another occasion. These findings are in line with Kleinman's suggestion that lay EMs are likely to be idiosyncratic and changeable, with Young's findings of fragmentary explanations borrowed from multiple sources, and with Helman's suggestion that lay theories of illness aetiology tend to be multi-causal. Even so, the plurality and the diversity of suggestions seem to be extraordinary. Hence, whereas the biomedical model declares TB to be an infectious disease caused by Mycobacterium Tuberculosis only, the Solubang thinking is wider and embraces a large number of possible causes: some traditional, some more contemporary.

Part of the explanation to the many diverse suggestions is likely found in the rather fluid situation in the country as a whole, including alterations in health care services on offer, the recent addition of biomedicine in particular. Another likely part is the common aptitude within a Hindu worldview to handle and harbour several explanations at one and the same time, the latter quite contrary to a common western urge, biomedicine included, for reduction and congruency.

A good portion of the most frequently suggested causes overlap with socio-biomedical reasoning of factors of importance for the development of the disease: a weak body; misuse of alcohol and smoking; poverty; lack of proper food. In the biomedical model, however, such features are not causal agents but conditions likely to compromise the immune system which gives the TB bacilli a chance to thrive and active TB to develop. Thus, that which appears 
to be common ground is not really so.

In a similar fashion, thoughts and ideas of what constitutes the disease are at a variance. To the biomedically trained person, TB is the proliferation of the TB bacilli within the patient's body. To the Solubang villagers, as to most Nepalis, 'tibi' is what is there to observe, the outward signs, such as the persistent coughing, the weakness, the lack of appetite, and so on. Even so, when suggesting the possible cause/s the focus is not on the symptoms per sebut on the person and his/her life circumstances. In Nepal, this is not a feature unique for TB but a common orientation in regard to misfortunes, ill-health included. In the terminology of Evans-Pritchard, the main causality emphasis is on the "why" aspects rather than on the "how" aspects. So, what about the most frequently mentioned causes in the interviews, "too much alcohol" and "a weak body"?

In connection with the start of anti-TB treatment, Solubang patients, as common all over Nepal, are told to abstain from alcohol and tobacco while on treatment and to eat nutritious food. Most Nepali patients are not likely to ask questions in health care situations and the mere fact of being told, "Do not drink alcohol!", "No tobacco!" and "Eat nutritious food!" has seemingly often been understood to mean that alcohol drinking, tobacco chewing/ smoking, and a lack of proper food are major causes for TB. Such an understanding has most likely been eased by ideas of this kind coinciding with traditional values and attitudes.

In traditional Hindu philosophy, in connection with the high castes, the drinking of alcohol is associated with ritual pollution which in turn is connected with negative conditions, ill-health included. The common suggestion of the alcohol intake being far too high in Solubang, a mainly high-caste community, is an ideological declaration of traditional and cosmic rules being broken with negative consequences for individuals and for the community. The same can be said about tobacco. Concurrently, excessive drinking was and is frowned upon, not for metaphysical reasons only but also for its observed harmful effects on hygiene, physical fitness, social relations and the household economy.

At the same time, it is and was obvious that not everyone drinking and smoking in excess ended up with TB. Asked why not, 
some replied that they did not know, while some suggested that most likely other causes were also involved. The reverse situation, that some persons not using alcohol and tobacco got TB, also prompted additional explanations.

To Solubang villagers "a weak body" has and has had a wider connotation than a strictly physical one. Constructed within an Ayurvedic and/or a Shamanistic framework, the expression signifies not only a physical entity but 'a person'. In this case, a person lacking the strength and qualities needed to withstand subversive and destructive influences. "A strong person", it was repeatedly emphasised, "will not get TB!"

"Poverty" was a cause mentioned by many. In biomedical rhetoric, TB is often referred to as 'a disease of poverty'. Some of the reasons for the latter coincide with those recognised by the Solubang inhabitants but others do not. Examples of features not recognised by Solubang residents but by western medicals are crowded living arrangements and insufficiently ventilated houses.

To the Solubang villagers, poverty signifies and signified worries, insufficient food, and exhausting work, an altogether stressful situation not conducive to happiness and good health. Moreover, it often meant that a family member had to go abroad, mostly to India. The suggestion that the cause of a person's TB maybe because "he worked in India", seemingly reflect sentiments and expectations of this kind. It also echoes a common idea of a person's constitution being adjusted to the place he/she belongs and when moved from his/her natural environment the person easily falls ill (Subedi 2001, p. 97).

The western view of the human body as neutral differs from the traditional Hindu/Buddhist outlook within which the basic attitude towards the body, as towards earthly existence as a whole, is negative. Bodily waste products defile. Heavy coughing, sputum, and blood, common in pulmonary TB, are abhorred. Pollution blackens and the statement, "the face of the TB patient is getting dark" is more likely a reflection of a general expectation of increased inner pollution manifesting itself in the hue of the skin than an observation of an actual change in skin colour. In advanced $\mathrm{TB}$, the patient will probably get paler rather than darker. 
The suggestion of TB as a "hereditary" disease is not about a shared genetic profile. In some interviews the concept referred to a shared Fate (Bhagya). In other interviews the expression simply referred to the transmittable nature of $\mathrm{TB}$, that is, to a more mundane process of disease transmission within a family. In yet others, there seemed to be a mixture of the two. It should be noted, that 'Karmic justice' was rarely spontaneously referred to by the respondents. Even so, it could be read between the lines in several replies, like in some 'hereditary' suggestions.

Salzberger, as mentioned, suggests that generally speaking, the responsibility for an illness is ascribed either to the victim or to external factors. In Solubang in regard to TB, the general tendency was for the responsibility to fall on the victim, sometimes directly, sometimes indirectly. This is in contrast to the biomedical model which depicts those affected as 'innocent victims' who happen to have been in the wrong place at the wrong time. At the same time, some of the fundamental Hindu concepts involved are extremely complex and multi-layered and do not easily fit into ready-made dichotomies. For instance, on one level, the idea of TB “...perhaps being the result of Solubang being in the shadow of a hill and therefore quite damp" is to ascribe the responsibility to external factors. On another level, the fact that a family is living under such negative circumstances is not.

"Because of bad Karma", as mentioned, was not a cause spontaneously referred to, albeit sometimes obviously lurking in the background. Fatalism was also in the picture, not necessarily as a primary driving force but as an explanation. This was clearly so when, in 2013, we asked the patients of the 2005 cohort if they thought that something could have been done to prevent them from getting the disease. The reply, with one exception, was a clear "No", and that even from those who had suggested that their TB was the result of too much drinking and smoking.

Foster and Anderson, as mentioned, propose a two-fold dichotomy of aetiologies: personalistic versus naturalistic aetiologies. In the two performed studies, TB in general was rarely suggested to be caused by an active, purposeful intervention of a sensate agent. Thus, 'tibi', in contrast to some of the other major 
diseases, leprosy and HIV/AIDS in particular, was not suggested to be "a curse by the gods" (it might have been in times past). This might be linked to TB, as of late, being perceived as a curable condition, 'a normal disease'. Even so, in specific cases, a few patients did include the possibility of an irritated god being in the picture, but never as the sole cause.

Parallel to the proposed cause/s there were uncertainties. The latter demonstrated in what was said but also in gesticulations: the many 'hola", 'maybes' and the frequent use of the Nepali gesture for "perhaps, who knows?". Part of the expressed uncertainty is likely to stem from an awareness of ideas and beliefs of old being questioned in the modern world but also to a common acceptance of reality being too complex to fathom.

In addition to expressed uncertainties there were also "I don't know" or "I really don't know". Whereas some of the "I don't know" replies were likely to be genuine, others might have been a strategy to avoid embarrassing suggestions. The latter is deduced from the fact that, in several of the families of those who said they did not know, there was or had been a TB patient and thus most likely some thoughts and ideas about what had brought about the disease. At the same time, however, concerted discussions about the reason/s for the TB in the family had, in several cases, evidently not taken place. The rationale for this might be traced to the notion of the less attention paid to a calamity the better, but also to a culturally imprinted hesitancy to point an accusing finger at someone, particularly at a relative. A hesitancy of this kind corresponds to members of households with a TB patient often emphasising that the patient him- or herself was the one to initiate the safety measures within the household. For the initiative to have come from someone else would have shamed the patient.

When directly asked, everyone, or almost everyone, as mentioned, readily agreed to TB being a transmittable disease yet spontaneously transmission as a cause was rarely mentioned. The latter may in part be connected with the previously mentioned unwillingness to point a finger at someone.

The idea of 'germs' and the mechanisms of transmissions as suggested in biomedical health messages are largely novel to 
adult Nepalis, particularly in the rural areas, and often not well understood. The belief of TB being transmittable, however, was and is not new. Within the Ayurvedic model all bodies, the human body included, are neither clearly edged nor sharply differentiated from their surroundings. The skin is a protective boundary but is not sealed (Marriot 1976). Cultural convictions and traditions about general infiltration and exchange of essence between those in contact with each other may well explain why separation measures were upheld among the members of TB households but less so with visitors from outside. The transmission of whatever is transmitted is not necessarily a quick process. For the absorption of a disease like $\mathrm{TB}$, prolonged intimacy is likely to be needed and thus an expected greater need for measures of separation for those continually rubbing shoulders than for casual visitors.

Within the homes, the precautionary measures taken coincided with traditionally expected routes for a transfer of ritual pollution: the sharing of what touches the mouth - the eating utensils; that which is next to the skin - used clothes; body to body - sleeping together (sexual intercourse definitely so); and the partaking of what has been on another person's plate (see below). Within traditional Nepali thinking each of these routes can be used positively to create equality and unity (shared pollution) but also negatively in an intended or unintended transfer of pollution. The same routes were obviously also believed to be crucial in a possible transfer of TB and thus the specific safety measures installed within the homes. At the same time, the charted routes were hardly relevant in the context of visitors' contact with a TB affected person inside or outside the home.

Concurrently, the biomedically proposed greatest danger, that is, the exposure and the inhalation of air-born droplets containing TB bacteria, was rarely in the calculation neither in regard to household members nor to outsiders. And yet, there were some remarks, not many but some, more so in 2013/14 than in 2005, about TB being transferred through "breathing", "sneezing", and "coughing". Rarely, if ever, however, were such suggestions linked with precautionary behaviour.

The separation measures carried out in regard to household 
members after the diagnosis might have had psychologically positive effects but are not likely to make or to have made an epidemiological difference as most transmissions within a household are likely to take place before the diagnosis. Post-diagnosis precautionary measures, according to the biomedical understanding, are primarily to protect causal contacts and so quite opposite to that which was and is taking place in Solubang and in large parts of Nepal. Moreover, the idea of the need for separate utensils, food, water, and clothes within the household does not square with the biomedical notion of human TB not being harmful from surfaces (Lamsal et al 2009:26). The precaution of not sharing a bed, including not having sexual intercourse, is more to the point, however, not because of the physical closeness per se but because of the short breathing distance in often badly ventilated sleeping quarters.

In Solubang as in most of Nepal, to eat the jutho, of a diseasepolluted person was and is not acceptable, neither in relation to family members nor to outsiders. In Solubang it was never mentioned as a cause of someone's TB. However, the many references to "not eating the jutho of a TB patient" and "TB patients not giving their jutho to anyone", testify to beliefs in its transmission potential. Not only is it a common idea among the population in general but also among health workers. Thus, several patients mentioned that health workers had advised them not to give their jutho to others. When asked why food leftovers are perceived as particularly dangerous the common reply was that whatever has been on a person's plate is likely to be soiled by the saliva of the person. From a traditional point of view saliva is discharged, concentrated, ritual pollution whereas from a biomedical point of view the same is germinfested secretion. In regard to the latter, the biomedical discourse, as mentioned, suggests that human TB bacilli are not transferred from surfaces: saliva, leftover food, and water included but only through air-born droplets, that is, by inhalation only. Even so, in both models, the mouth is a key gate for the transfer of the disease but quite differently so. The situation of precautionary initiatives within TB households being upheld far longer than needed, that is, long after a patient is non-infectious, is at least in part the result of health workers not putting all that much effort into explaining 
the ins and outs of the disease. There is no need for separation measures in extra-pulmonary cases and in initially infectious cases not after a sputum conversion. The latter is commonly determined by routine microscopic examination of sputum after the first two months of medication. In part, however, the prolongation of the safety measures within the households was also prompted by the 'mystery' of why medicines are needed many months after the disappearance of signs and symptoms.

The one thing hammered in by the health workers is the absolute 'must' to complete the full course of anti-TB medicines. And, the patients comply, partly because they are aware of their own incomplete understanding of the disease and partly not to kindle the anger of the health workers. Moreover, the order to continue with the medicines, even after the regaining of health (subjectively), must mean, it is thought, that within the body there are remnants of the disease and thus it is reasoned, most likely also a need for safety measures for at least as long as the treatment is going on and, according to some, the longer the better.

\section{Conclusion}

In times past, recognised TB was a fatal and an utterly feared disease and rightly so. The conditions denoted 'khapte', 'sukenashak' and later 'kshayarog' were likely to be pulmonary $\mathrm{TB}$ in advanced stages and likely to soon end in death. The combination of fear of a transmission with fear of death-pollution might well have been the major reason why, in former days, persons with advanced TB were put out of the house. The arrival of the biomedical anti-TB medicines has drastically changed the situation for the better and is widely recognised to have done so. Thus, the common understanding in Solubang, as in Nepal as a whole, is that the best and perhaps the only effective treatment for TB is within the biomedical sector and this irrespective of what has caused the disease. Even so, presumed causes influence what is taking place in the lives of the patients and their families before, during, and sometimes even after treatment. Thus, in order to provide as good a support as possible, health workers engaged in the treatment process need to have an understanding not of the biomedical model 
of TB only but also of the explanatory models of the patients and their communities.

\section{Notes:}

${ }^{1}$ The National Tuberculosis Centre's web site, October 2015.

${ }^{2}$ Approvals for the research were granted by the Nepal Health Research Council. The formal interviews took place after informed consent. The research was mainly financed by a grant from the Swedish Research Council. Most of the field work was carried out by Mr Bishnu Acharya, an employee of the International Nepal Fellowship (the government's main counterpart in TB work in the Mid-Western Development Region).

\section{References}

Baral, S.C. et al (2011). Knowledge, attitude and practice (KAP) on Tuberculosis (TB) and co-infection of TB and HIV in Nepal. Kathmandu: Health Research and Social Development Forum (HERD).

Bhatt, C.P. et al. (2009). Nepalese people's knowledge about Tuberculosis. SAARC Journal of Tuberculosis, Lung Diseases and HIV/AIDS, 6(2), 31-37.

Dheda, K. et al. (2010). The immunology of tuberculosis: From bench to bedside. Respirology, 15(3), 433-450.

Evans-Pritchard, E.E. (1937). Witchcraft, oracles and magic among the Azande. 1960 edition. Oxford: The Clarendon Press.

Foster, G.M. and Anderson, B.G. (1978). Medical anthropology. New York: John Wiley and Sons.

Helman, C. (2007). Culture, Health and Illness. 5th edition. London: Hodder Arnold.

Kleinman, A. (1980). Patients and healers in the context of culture. Berkley: University Press.

Lamsal DK, Lewis OD, Smith S, \& Jha N. (2009). Factors related to defaulters and treatment failure of tuberculosis patients in the DOTS program in the Sunsari district of Eastern Nepal. SAARC Journal of Tuberculosis, Lung Diseases and HIV/AIDS, 6(1), 25-30.

Mariott, M. (1976). Hindu transactions: Diversity without dualism. 
In Transaction and Meaning B. Kapferer (Ed.). Philadelphia: Institute for study of human issues.

Salzberger, R.C. (1976). Cancer: Assumptions and reality concerning delay, ignorance and fear. In J. B. Loudon (ed.), Social anthropology and medicine (pp. 150-189). New York: Academic Press.

Subedi, M.S. (2001). Medical anthropology of Nepal. Kathmandu: Udaya Books.

Young, A. (1992). The anthropology of illness and sickness. Annual Review of Anthropology, 11, 257-285. 